\title{
The Lorentz force in atmospheres of chemically peculiar stars: 56 Arietis
}

\author{
D. Shulyak ${ }^{1}$, O. Kochukhov ${ }^{2}$, G. Valyavin ${ }^{3}$, B.-C. Lee ${ }^{4}$, G. Galazutdinov ${ }^{5}$, K.-M. Kim ${ }^{4}$, \\ I. $\mathrm{Han}^{4}$, and T. Burlakova ${ }^{6}$
}

1 Institut für Astronomie, Universität Wien, Türkenschanzstraße 17, 1180 Wien, Austria e-mail: denis.shulyak@gmail.com

2 Department of Physics and Astronomy, Uppsala University, Box 515, 751 20, Uppsala, Sweden

3 Observatorio Astronómico Nacional SPM, Instituto de Astronomía, Universidad Nacional Autónoma de México, Ensenada, BC, México

${ }^{4}$ Korea Astronomy and Space Science Institute, 61-1, Whaam-Dong, Youseong-Gu, Taejeon, 305-348, Rep. of Korea

5 Department of Physics and Astronomy, Seoul National University, Gwanak-gu, Seoul 151-747, Rep. of Korea

${ }^{6}$ Special Astrophysical Observatory, Russian Academy of Sciences, Nizhnii Arkhyz, Karachai Cherkess Republic, 369167, Russia

Received 2 June 2009 / Accepted 1 October 2009

\section{ABSTRACT}

\begin{abstract}
Context. The presence of electric currents in the atmospheres of magnetic chemically peculiar $(\mathrm{mCP})$ stars could theoretically constrain the nature and evolution of magnetic field in these stars. The Lorentz force, which is the result of the interaction between the magnetic field and the induced currents, modifies the atmospheric structure and induces characteristic rotational variability of pressure-sensitive spectroscopic features, which can be analyzed using phase-resolved spectroscopic observations.

Aims. In this work we continue presenting the results of the magnetic pressure studies in mCP stars focusing on the high-resolution spectroscopic observations of Bp star 56 Ari.

Methods. We interpreted observations in the framework of the model atmosphere analyzis, which accounts for the Lorentz force effects. We used the LLmodels stellar model atmosphere code for the calculation of the magnetic pressure effects in the atmosphere of 56 Ari by taking the realistic chemistry of the star and accurate computations of the microscopic plasma properties into account. The SYNTH 3 code was employed to simulate phase-resolved variability of Balmer lines.

Results. We detected a significant variability of the $\mathrm{H} \alpha, \mathrm{H} \beta$, and $\mathrm{H} \gamma$ spectral lines during a full rotation cycle of the star. We demonstrate that the model with the outward-directed Lorentz force in the dipole+quadrupole configuration is likely to reproduce the observed hydrogen lines variation. These results present strong evidence of non-zero global electric currents in the atmosphere of this early-type magnetic star.
\end{abstract}

Key words. stars: chemically peculiar - stars: magnetic field - stars: individual: 56 Ari - stars: atmospheres

\section{Introduction}

The atmospheres of magnetic chemically peculiar (mCP) stars display the presence of global magnetic fields ranging in strength from a few hundred $\mathrm{G}$ up to several tens of $\mathrm{kG}$ (Landstreet 2001), with the global configurations well represented by dipolar or low-order multipolar components (Bagnulo et al. 2002) that are likely stable during significant time intervals. The stability of the atmospheres against strong convective motions and the large-scale magnetic fields provide unique conditions for studing the secular evolution of global cosmic magnetic fields and other dynamical processes that may take place in the magnetized plasma. In particular, the slow variation of the field geometry and strength changes the pressure-force balance in the atmosphere via the induced Lorentz force, which makes it possible to detect it observationally and establish a number of important constraints on the plausible scenarios of the magnetic field evolution in early-type stars.

Among the known characteristics of $\mathrm{mCP}$ stars, the variability of hydrogen Balmer lines is poorly understood. For some of stars it can be connected to the inhomogeneous surface distribution of chemical elements, possible temperature variations, and/or stellar rotation (see, for example, discussion in Lehmann et al. 2007). At the same time, some of the magnetic stars demonstrate the characteristic shape of the Balmer line variability that cannot be simply described by temperature or abundance effects. For example, Kroll (1989) showed that at least part of the variability detected in several $\mathrm{mCP}$ stars can be attributed to the pressure effects, indicating a non-zero Lorentz force in their atmospheres.

Different atmosphere models with the Lorenz force were considered by several authors (see review in Valyavin et al. 2004). In this study we follow approaches by Valyavin et al. (2004) considering the problem in terms of induced atmospheric electric currents interacting with magnetic fields. The authors predict that the amplitude of the variations in hydrogen Balmer lines seen in real stars can be described if one assumes strong electric currents flowing in upper atmospheric layers of these objects. Later on, Shulyak et al. (2007, Paper I hereafter) extended and improved this model to more complicated field geometries and accurate treatment of magnetized plasma properties. Their first direct implementation of new model atmospheres to the analyzis of the hydrogen spectra of one of the brightest mCP star $\theta$ Aur shows that their rotational modulation can be induced by the Lorentz force effect, which is not directly connected to the temperature or abundance variation across the stellar surface. The precise analyzis of the longitudinal magnetic field variation 
and subsequent modeling of the magnetic pressure for every observed rotational phase of the star allowed us to constrain the magnitude and the direction of the Lorentz force. In particular, the outward-directed Lorentz force (i.e. directed outside the stellar interior along radius) was found to provide the best fit to observations, in combination with rather strong induced e.m.f. (electro-magnetic force) of about $1 \times 10^{-11} \mathrm{cgs}$ units, which was found to play an important role in the overall hydrostatic structure of the stellar atmosphere.

The knowledge of the direction and the strength of the Lorentz force is important for understanding of physical mechanisms that are responsible for such strong surface currents and their interaction with global, large-scale magnetic fields (see discussion in Paper I for more details). Taking this into account and following the pioneering work by Kroll (1989), we initiated a new spectroscopic search of hydrogen line variability in a number of magnetic main-sequence stars (Valyavin et al. 2005).

In this paper we present the phase-resolved high-resolution observations of one of the weak-field $\left(\left|\left\langle B_{z}\right\rangle\right|<500 \mathrm{G}\right) \mathrm{mCP}$ star 56 Ari (HD 19832). We detected significant variation in the Balmer line profiles and interpreted it in terms of the non-forcefree magnetic field configuration.

The overview of observations will be presented in the next section. Then, in Sect. 3 we give a short description of the model used to simulate the effects of the magnetic pressure. The main results are summarized in Sect. 4 with conclusions and discussion given in Sects. 5 and 6 respectively.

\section{Observations}

Observations of 56 Ari were carried out with the BOES echelle spectrograph installed at the $1.8 \mathrm{~m}$ telescope of the Korean Astronomy and Space Science Institute. The spectrograph and observational procedures are described by Kim et al. (2000, 2007). The instrument is a moderate-beam, fiber-fed highresolution spectrometer that incorporates 3 STU polymicro fibers of 300,200 , and $80 \mu \mathrm{m}$ core diameters (corresponding spectral resolutions are $\lambda / \Delta \lambda=30000,45000$, and 90000 , respectively). The medium-resolution mode was employed in the present study. The working wavelength range is from $3500 \AA$ to $10000 \AA$.

Seventeen spectra of the star were recorded in the course of 10 observing nights from 2004 to 2006 . Typical exposure times of a few minutes allowed $S / N \approx 250-300$ to be achieved. Table 1 gives an overview of our observations. Throughout this study we implement the ephemeris derived by Adelman et al. (2001) using linear changing period model:

$\mathrm{JD}=2434322.354+\frac{0.7278883}{1+0.7278883 \cdot S \cdot\left(t-t_{0}\right)}$,

where $t_{0}=2434322.354$ and $S=-1.35 \times 10^{-9}$. Details related to spectral data reduction and processing, and a study of the spectrograph's stability are presented in Shulyak et al. (2007), and are not described here. The accuracy of the continuum normalization around Balmer lines is estimated to be approximately $0.2-0.3 \%$.

\section{Model}

\subsection{General equations and approximations}

In this section we follow the approach and methods outlined previously in Paper I. However, for the sake of explanation, we find
Table 1. Observations of 56 Ari.

\begin{tabular}{ccc}
\hline \hline No. & JD & Rotation phase \\
\hline 1 & 2453250.2191 & 0.321 \\
2 & 2453250.3017 & 0.435 \\
3 & 2453251.2284 & 0.708 \\
4 & 2453251.3359 & 0.855 \\
5 & 2453306.1282 & 0.128 \\
6 & 2453306.2942 & 0.356 \\
7 & 2453308.0681 & 0.793 \\
8 & 2453309.0295 & 0.114 \\
9 & 2453309.1620 & 0.296 \\
10 & 2453666.0880 & 0.636 \\
11 & 2453671.0690 & 0.478 \\
12 & 2453671.0860 & 0.502 \\
13 & 2453759.0098 & 0.290 \\
14 & 2453760.0284 & 0.689 \\
15 & 2453760.9714 & 0.985 \\
16 & 2453762.0026 & 0.402 \\
17 & 2453762.9682 & 0.728 \\
\hline
\end{tabular}

it useful to state here some of the general assumptions used in the modeling procedure:

1. the stellar surface magnetic field is axisymmetric and is dominated by dipolar or dipole+quadrupolar component in all atmospheric layers;

2. the induced e.m.f. has only an azimuthal component, similar to that described by Wrubel (1952), who considered decay of the global stellar magnetic field. In this case the distribution of the surface electric currents can be expressed by the Legendre polynomials $P_{n}^{1}(\mu)$, where $n=1$ for dipole, $n=2$ for quadrupole, etc., and $\mu=\cos \theta$ is the cosine of the colatitude angle $\theta$ which is counted in the coordinate system connected to the symmetry axis of the magnetic field;

3 . the atmospheric layers are assumed to be in static equilibrium and no horizontal motions are present;

4. stellar rotation, Hall's currents, ambipolar diffusion and other dynamical processes are neglected.

Taking these approximations into account and using Maxwell equation for field vectors and Ohm's law, one can write the hydrostatic equation in the form

$\frac{\partial P_{\text {total }}}{\partial r}=-\rho g \pm \frac{1}{c} \lambda_{\perp} \sum_{n} c_{n} P_{n}^{1}(\mu) \sum_{n} B_{\theta}^{(n)}=-\rho g_{\mathrm{eff}}$.

Obtaining this equation we used the superposition principle for field vectors and the solution of Maxwell equations for each of the multipolar components following Wrubel (1952). We also suppose that $\boldsymbol{E} \perp \boldsymbol{B}$. Here $c_{n}$ represents the effective electric field generated by the $n$-th magnetic field component at the stellar magnetic equator and $B_{\theta}$ is the horizontal field component. The signs "+" and "-" refer to the outward- and inward-directed Lorentz forces, respectively.

We note that the values of $c_{n}$ are free parameters to be found by using our model. These values represent the fundamental characteristics that can be used for building self-consistent models of the global stellar magnetic field geometry and its evolution. Thus, an indirect measurement of these parameters via the study of the Lorentz force is of fundamental importance for understanding the stellar magnetism.

Calculation of the electric conductivity $\lambda_{\perp}$ is carried out using the Lorentz collision model where only binary collisions between particles are allowed which is a good approximation for a 
Table 2. Abundances (in $\log N_{\mathrm{el}} / N_{\text {total }}$ ) of 56 Ari, used for determining model atmosphere parameters.

\begin{tabular}{cccccc}
\hline \hline & $\mathrm{He}$ & $\mathrm{Mg}$ & $\mathrm{Al}$ & $\mathrm{Si}$ & $\mathrm{Fe}$ \\
\hline 56 Ari & -2.10 & -5.51 & -6.17 & -3.53 & -4.09 \\
Sun & -1.10 & -4.51 & -5.67 & -4.53 & -4.59 \\
\hline
\end{tabular}

low density stellar atmosphere plasma. The detailed description and basic relationships of this approach are given in Paper I.

From Eq. (2) it is seen that, in the presence of electric currents and magnetic field, the rotation of a star can produce phasedependent Lorentz-force term (due to a variation of $B_{\theta}^{(n)}$ and $\lambda_{\perp}$ ), which will in turn modify the hydrostatic structure of the atmosphere, manifesting itself as a variation of pressure-sensitive spectral lines.

\subsection{Model atmospheres with Lorentz force}

Our calculations were carried out with the stellar model atmosphere code LLmodels developed by Shulyak et al. (2004). At each iteration the code calculates electric conductivity in all atmospheric layers using all available charged and neutral plasma particles. The conductivity is then used to evaluate the magnetic contribution to the magnetic gravity and to execute temperature and mass correction procedure.

The input parameters for the calculation of magnetic pressure are the direction of the Lorentz force (inward- or outwarddirected), e.m.f. at the stellar equator, mean surface magnetic field modulus $\langle\boldsymbol{B}\rangle$, and the product of the two sums in Eq. (2) containing contribution from all considered multipolar components for every single rotational phase of the star.

As can be seen from Eq. (2), there is some critical value of $c_{n}$ that may produce unstable solution in the case of the outwarddirected Lorentz force. Such models cannot be considered in the hydrostatic equilibrium approximation introduced above and were deemed non-physical in our calculations. Thus, for each set of models, we restricted $c_{n}$ values to ensure static equilibrium.

The atomic line list was extracted from the VALD database (Piskunov et al. 1995; Kupka et al. 1999), including all lines originating from the predicted and observed energy levels. This line list was used as input for the lines opacity calculation in the LLmodels code.

\section{Numerical results}

\subsection{Model atmosphere parameters of 56 Ari}

The model atmosphere parameters, $\log (g)$ and $T_{\text {eff }}$, were determined using theoretical fit of the hydrogen Balmer lines and spectral energy distribution. It was constructed by combining the average of the optical spectrophotometric scans obtained by Adelman (1983) and low dispersion UV spectrograms from the IUE INES ${ }^{1}$ database. For modeling the hydrogen $\mathrm{H} \beta$ and $\mathrm{H} \gamma$ line profiles we used the mean spectrum of 56 Ari averaged other all 17 observed rotational phases. The projected rotational velocity $v \sin i=160 \mathrm{~km} \mathrm{~s}^{-1}$ was taken from Hatzes (1993). Ryabchikova (2003) used the same value in a more recent Doppler imaging study. Individual abundances of several chemical elements, listed in Table 2, were determined as described below (Sect. 4.3).

Synthetic Balmer line profiles were calculated using the SYNTH3 program (Kochukhov 2007), which incorporates

\footnotetext{
1 http://ines.ts.astro.it/
}

recent improvements in the treatment of the hydrogen line opacity (Barklem et al. 2000). The stellar energy distribution and Balmer lines are approximated best with the following parameters: $T_{\text {eff }}=12800 \pm 300 \mathrm{~K}, \log (g)=4.0 \pm 0.05$. Note that such high accuracy of the determined parameters is just an internal accuracy obtained from our technique, which we used to fit the data. Real parameters may be slightly different from the obtained ones due to various systematic error sources, but this does not play a significant role in our study.

Comparisons of the observations and model predictions are presented in Figs. 1 and 2. We transformed Adelman's spectrophotometric observations to the absolute units following Lipski \& Stępień (2008). Since the absolute calibration of IUE fluxes around their red end have substantial uncertainties (see Lipski \& Stępień 2008), we scaled the IUE fluxes by $\approx 10 \%$ to match the Adelman's data in the near-UV region. This correction is comparable to the offset between alternative flux calibrations suggested for the IUE data (García-Gil et al. 2005). Applying the correction ensured that the IUE spectra are smooth continuation of the optical spectrophotometry. Then the theoretical fluxes can be adjusted to fit the combined set of observations. Lipski \& Stępień (2008) also noted the discrepancy between observations in visual and UV, but did not do any attempts to correct it. This is why their final effective temperature of 56 Ari was found to be $T_{\text {eff }}=12250 \mathrm{~K}$ resulting from the fact that ignoring the obviously spurious offset between the observed datasets one needs a lower $T_{\text {eff }}$ to fit the IUE fluxes simultaneously with Adelman's fluxes redward of the Balmer jump. As an example we also show in Fig. 1 theoretical flux obtained from the $T_{\text {eff }}=12300 \mathrm{~K}$, $\log (g)=3.9$ model which fits reasonably well the hydrogen Balmer lines (see Fig. 2), but fails to reproduce either Adelman's or IUE data.

Photometric observations in the Strömgren and UBV systems also point to the higher $T_{\text {eff }}$ and $\log (g)$ of 56 Ari. For instance, comparing theoretically computed color-indices with observations of Hauck \& Mermilliod (1998) and Nicolet (1978) we find that observed photometric parameters $(b-y=-0.052$, $\left.c_{1}=0.55, B-V=-0.12, U-B=-0.42\right)$ are best fitted with $T_{\text {eff }}=12800 \mathrm{~K}, \log (g)=4.0$ model $(b-y=-0.052$, $\left.c_{1}=0.60, B-V=-0.12, U-B=-0.43\right)$ rather than more cooler $T_{\text {eff }}=12300 \mathrm{~K}, \log (g)=3.9$ model $(b-y=-0.048$, $\left.c_{1}=0.67, B-V=-0.12, U-B=-0.38\right)$.

Finally, recent studies by Kochukhov et al. (2005) and Khan \& Shulyak (2006) showed that the effects of Zeeman splitting and polarized radiative transfer on the model atmosphere structure and shapes of hydrogen line profiles are less than $0.1 \%$ for magnetic field intensities around $1 \mathrm{kG}$, so they can be safely neglected in the present investigation.

\subsection{Magnetic-field geometry}

To calculate the Lorentz force effects, it is essential to specify the magnetic-field geometry (see Eq. (2)). For this purpose we made use of the longitudinal magnetic field measurements obtained by Borra $\&$ Landstreet (1980) using $\mathrm{H} \beta$ photopolarimetric technique. The authors observed a smooth single-wave $\left\langle B_{z}\right\rangle$ variation with rotation phase and concluded that it is probably caused by a dipole inclined to the rotation axis of the star.

Generalizing this work, we have approximated the magneticfield topology of 56 Ari by a combination of the dipole and axisymmetric quadrupole magnetic components. We also assumed that the symmetry axes of the dipole and quadrupole magnetic fields are parallel. Thus, the magnetic model parameters include the polar strength of the dipolar component $B_{\mathrm{d}}$, relative 


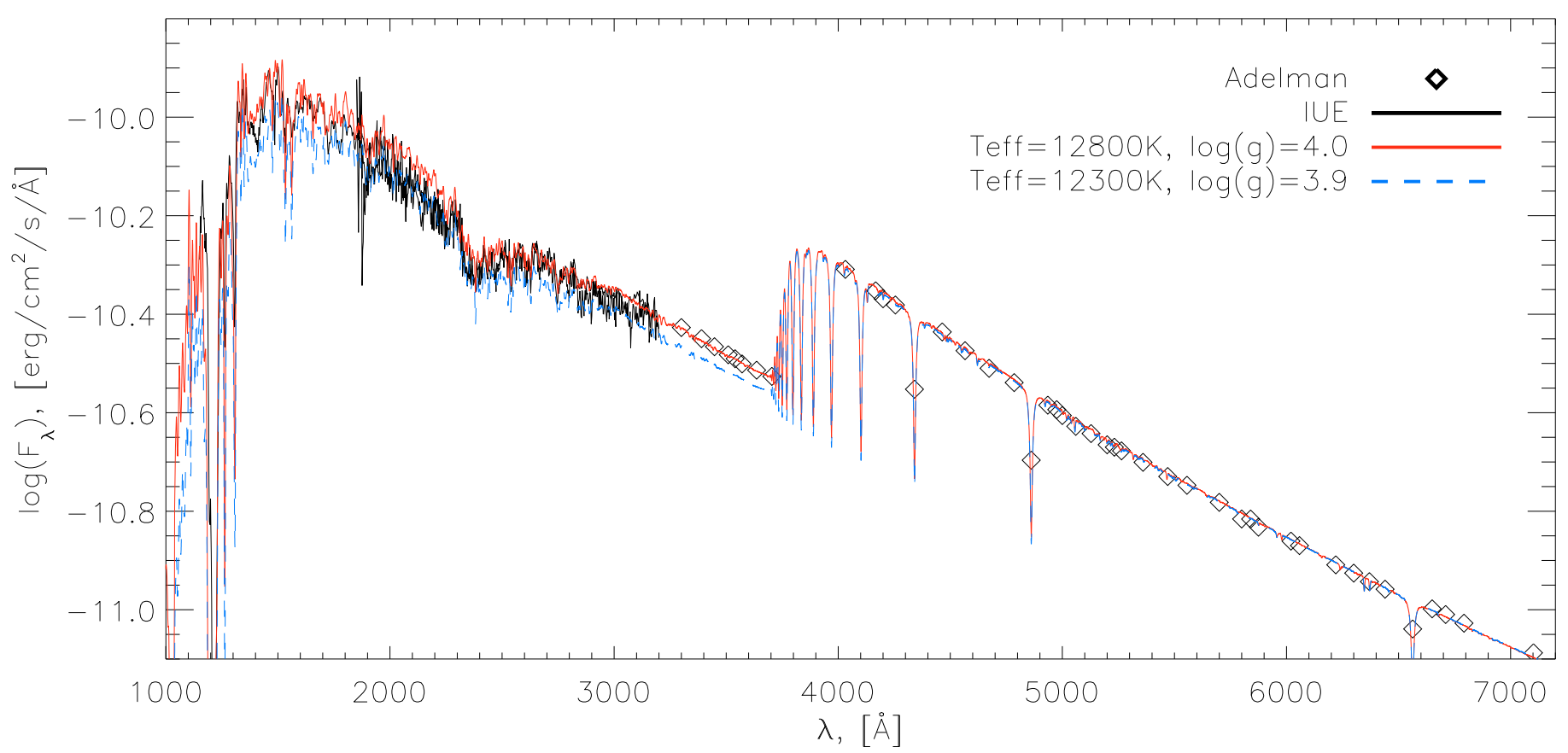

Fig. 1. Comparison of the observed and computed spectral energy distributions of 56 Ari. Theoretical models correspond to $T_{\text {eff }}=12300 \mathrm{~K}$, $\log (g)=3.9$ and $T_{\text {eff }}=12800 \mathrm{~K}, \log (g)=4.0$. The model fluxes have been convolved with an $F W H M=10 \AA$ Gaussian kernel for a better view.

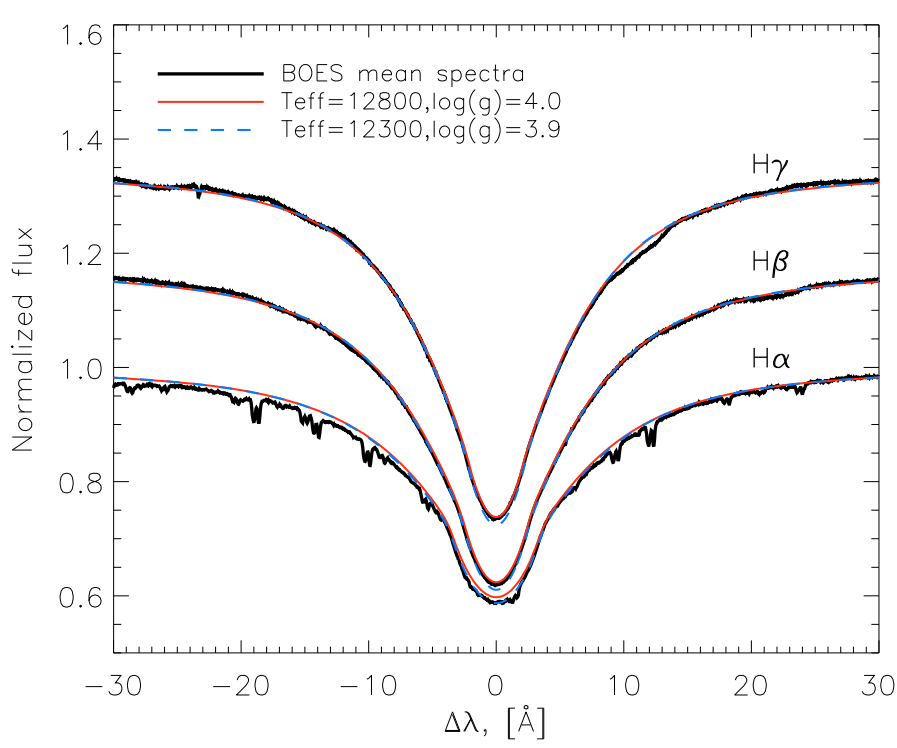

Fig. 2. Comparison of the observed and computed $\mathrm{H} \alpha, \mathrm{H} \beta$, and $\mathrm{H} \gamma$ line profiles.

contribution of the quadrupole field $B_{\mathrm{q}} / B_{\mathrm{d}}$, magnetic obliquity $\beta$, and inclination angle $i$ of the stellar rotation axis with respect to the line of sight. The last parameter can be estimated from the usual oblique rotator relation connecting stellar radius, rotation period, and $v \sin i$. Employing the recently revised Hipparcos parallax of 56 Ari, $\pi=6.49 \pm 0.76$ mas (van Leeuwen 2007), $T_{\text {eff }}=12800 \mathrm{~K}$ and bolometric correction $B C=-0.74$ determined by Lipski \& Stępień (2008), we found a stellar radius $R=2.8 \pm 0.4 R_{\odot}$ and inclination angle $i=57 \pm 13^{\circ}$. However, the previous Doppler imaging studies of Hatzes (1993) and Ryabchikova (2003) favored the value of $i=70^{\circ}$. Consequently we decided to explore the model parameters for the entire range of $i=50-70^{\circ}$.

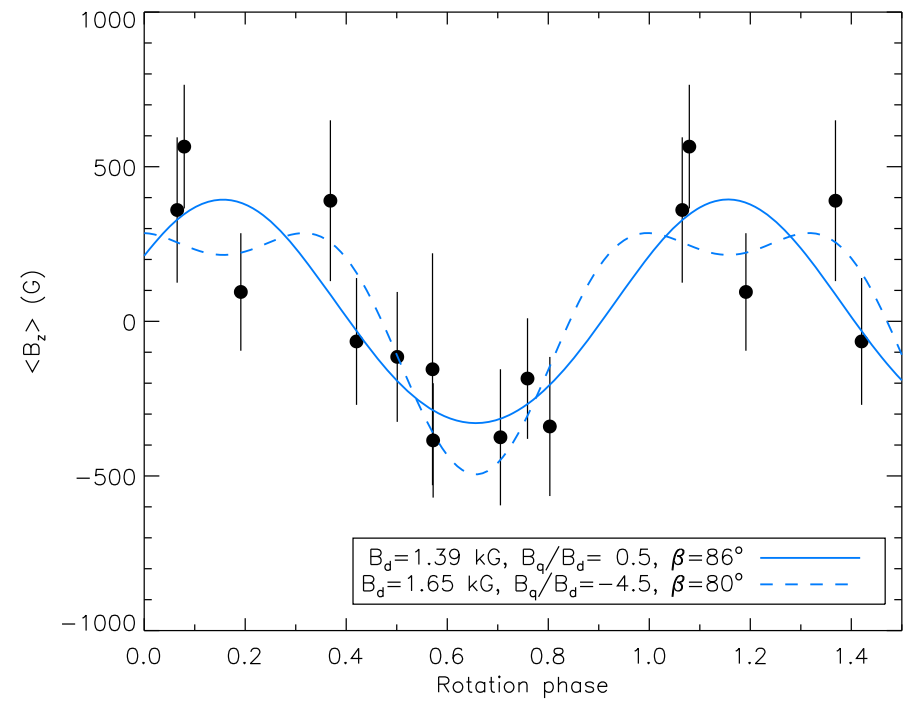

Fig. 3. Comparison of the longitudinal field observations of 56 Ari (symbols) and the model curves for $i=60^{\circ}$ and two extreme values of the $B_{\mathrm{q}} / B_{\mathrm{d}}$ parameter (lines).

The remaining free parameters of the magnetic field model were determined with the least-square fit of the observed $\left\langle B_{z}\right\rangle$ variation (Borra \& Landstreet 1980). Compared to $\theta$ Aur investigated in Paper I, the longitudinal magnetic field curve of 56 Ari is poorly defined due to large observational errors and a relatively small number of measurements. In this situation the acceptable solutions for the magnetic field geometry fall in a rather wide range: from $B_{\mathrm{q}} / B_{\mathrm{d}}=0.5$ to $B_{\mathrm{q}} / B_{\mathrm{d}}=-5$ for $i=50^{\circ}$, to $B_{\mathrm{q}} / B_{\mathrm{d}}=-4.5$ for $i=60^{\circ}$, and to $B_{\mathrm{q}} / B_{\mathrm{d}}=-4$ for $i=70^{\circ}$ (Fig. 3). The corresponding dipolar field strength range is $B_{\mathrm{d}}=1.3-1.8 \mathrm{kG}$ and magnetic obliquity is $\beta=70-90^{\circ}$. The lowest $\chi_{v}^{2} \equiv \chi^{2} / v$ was found between $B_{\mathrm{q}} / B_{\mathrm{d}}=[-1.5,-3]$ with the average value $\chi_{v}^{2} \approx 0.66$ while $\chi_{v}^{2} \approx 0.71$ for other 
geometries: with such a small difference we conclude that all the considered magnetic field geometries are equally possible.

\subsection{Effects of horizontal abundance distribution}

We used spectrum synthesis calculations to access chemical properties of the atmosphere of 56 Ari. Abundances of $\mathrm{He}, \mathrm{Al}$, $\mathrm{Mg}, \mathrm{Si}$, and $\mathrm{Fe}$ were determined by fitting SYNTH3 spectra to the average observations of the star. Results of this analyzis, summarized in Table 2, indicate that $\mathrm{He}, \mathrm{Al}$ and $\mathrm{Mg}$ are deficient in the atmosphere of 56 Ari with respect to the solar chemical composition. Fe is moderately overabundant while $\mathrm{Si}$ is strongly enhanced. Abundance of $\mathrm{Cr}$ cannot be determined reliably, but since no prominent $\mathrm{Cr}$ II lines are present in the spectrum of 56 Ari, we concluded that its overabundance is most likely smaller than that of Fe.

In addition to the mean abundances analyzis we interpreted variation in the phase-resolved spectra using the Doppler imaging (DI) technique (Kochukhov et al. 2004). Details of this work will be presented in a separate publication. here we are only concerned with the possible effect of the horizontal abundance variations on the hydrogen line profiles. Effects of the possible vertical stratitification of chemical elemets are ignored because, for the purpose of our study, they will not differ much from the effects of horizontal abundance inhomogeneities. By deriving surface-resolved abundances, we effectively account for the line opacity variation that would be introduced by chemical stratification.

Among the elements mentioned above, only $\mathrm{Mg}$ and $\mathrm{Si}$ show strong line profile variations. Therefore, we reconstructed abundance maps of Si using the red doublets Si II 6347, $6371 \AA$, and $\mathrm{Mg}$ using the $4481 \AA$ line. The modeling of the last region also included the He I $4471 \AA$ line, which allowed us to estimate potential influence of the He abundance variation on the model atmosphere structure and hydrogen line profile behavior.

Analyzis of $\mathrm{Si}, \mathrm{He}$, and $\mathrm{Mg}$ demonstrated that horizontal abundance inhomogeneities give a negligible contribution to the hydrogen line profile variation. We computed model atmospheres with the surface-averaged abundances for each rotational phase of the star. Figure 4 shows that the resulting standard deviation of the synthetic profiles is minute compared to the variation observed in the line wings of $\mathrm{H} \alpha, \mathrm{H} \beta$, and $\mathrm{H} \gamma$.

Much larger abundance gradients of the iron peak elements are required to induce a noticeable modulation of the hydrogen line profiles during rotation cycle. Figure 4 shows results of the numerical experiment where we assumed that Fe has the same horizontal distribution as Si but with 10 times higher contrast. This calculation contradicts the actual observations, since the Fe lines in 56 Ari vary weakly and differently from $\mathrm{Si}$, but it represents a useful illustration of the impact of chemical inhomogeneities. One can see that the metal abundance spots lead to the variation in the depth of hydrogen line cores, while in 56 Ari we see changes in the line wings. Thus, following Paper I and Kroll (1989), we are led to conclude that the chemical spots cannot contribute to the observed Balmer line wing variations.

\subsection{The Lorentz force}

To predict the phase-resolved variability in hydrogen line profiles, both the inward- and outward-directed Lorentz forces are examined through the model atmosphere calculations. The actual magnetic input parameters of computations with the LLmodels code include the sign of the Lorentz force, magnetic

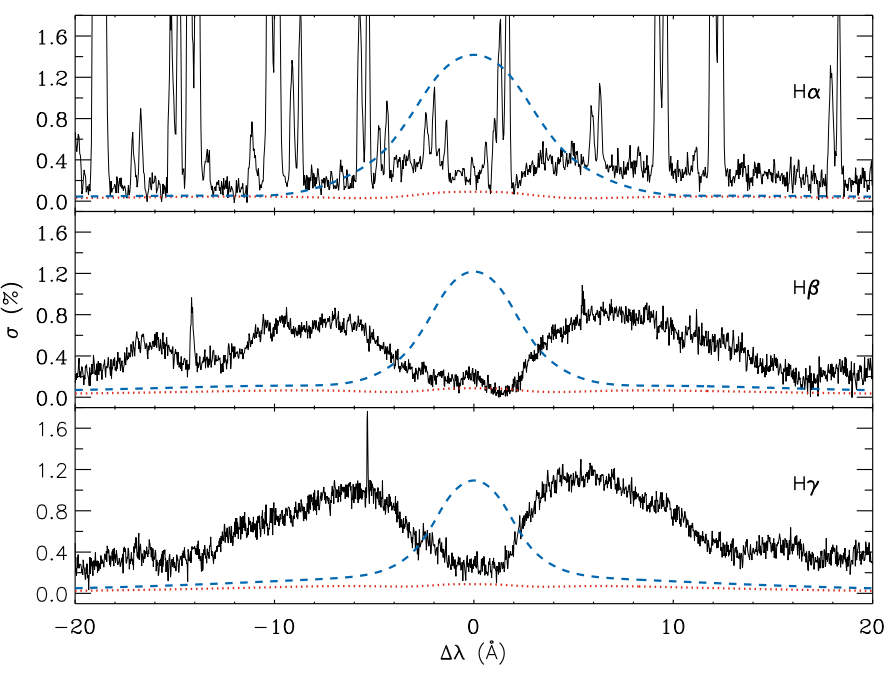

Fig. 4. The standard deviation $\sigma$ at the $\mathrm{H} \alpha, \mathrm{H} \beta$, and $\mathrm{H} \gamma$ lines. Observations are shown by solid lines. Dotted line - variability due to inhomogeneous element distributions in 56 Ari. The additional effect of the hypothetical extreme horizontal Fe gradient is shown by the dashed line (see text for explanations).

field modulus $B$, and the product of two sums $\sum c_{n} P_{n}^{1} \sum B_{\theta}^{(n)}$ (see Eq. (2)). We take the last two parameters to be disk-averaged at the individual rotation phases incorporating them in 1D stellar atmosphere code. The corresponding phase curves of the magnetic parameters relevant to our calculations are illustrated in Fig. 6.

By taking the wide variety of possible solutions for the surface magnetic-field geometry into account (see Sect. 4.2), we calculated several model grids with the outward- and inwarddirected Lorentz force and $B_{\mathrm{q}} / B_{\mathrm{d}}$ ranging from 0.5 to -4.5 with a step of 0.5 . The calculations show that, in order to reproduce the amplitudes of the observed standard deviations due to the profile variations, the effective electric field should be in the range $c_{1}=1 \times 10^{-10}-5 \times 10^{-10} \mathrm{cgs}$ in the case of the inward-directed Lorentz force and $c_{1}=1.2 \times 10^{-11}-1 \times 10^{-10} \mathrm{cgs}$ in the case of the outward-directed Lorentz force. Under the assumption of a purely dipolar configuration, these values are: $c_{1}=5 \times 10^{-10} \mathrm{cgs}$ for inward-directed and $c_{1}=7.5 \times 10^{-11} \mathrm{cgs}$ for outward-directed Lorentz forces.

It was not possible to fit the amplitude of the observed standard deviation with the inward-directed Lorentz force for all models considered in our study. This is true for models with $B_{\mathrm{q}} / B_{\mathrm{d}}>-1$, where the changes in longitudinal magnetic field and magnetic field modulus result in very narrow phase-resolved variations in the magnetic force term. As an example, the left panel of Fig. 5 illustrates the run of the $g_{\text {eff }}$ with the Rosseland optical depth in the atmosphere of 56 Ari $\left(B_{\mathrm{q}} / B_{\mathrm{d}}=-2.5\right)$ for inward- and outward directed Lorentz forces computed under different assumptions about the induced electric field. An increase in the e.m.f. value by a factor of two considerably changes the amplitude of the effective gravity, but the computed standard deviation does not change much (see lower left panel of Fig. 5). This is why even a large increase of e.m.f. does not show up itself in a standard deviation plot. Obviously, the situation may change once a more complex geometry of the magnetic field is introduced; however, it is connected with the introduction of additional free parameters making the fitting procedure ambiguous. In contrast, the outward-directed magnetic force seems to have a greater impact on the model pressure structure: varying e.m.f. value from $1 \times 10^{-11} \mathrm{cgs}$ to $1.2 \times 10^{-11} \mathrm{cgs}$ changes 

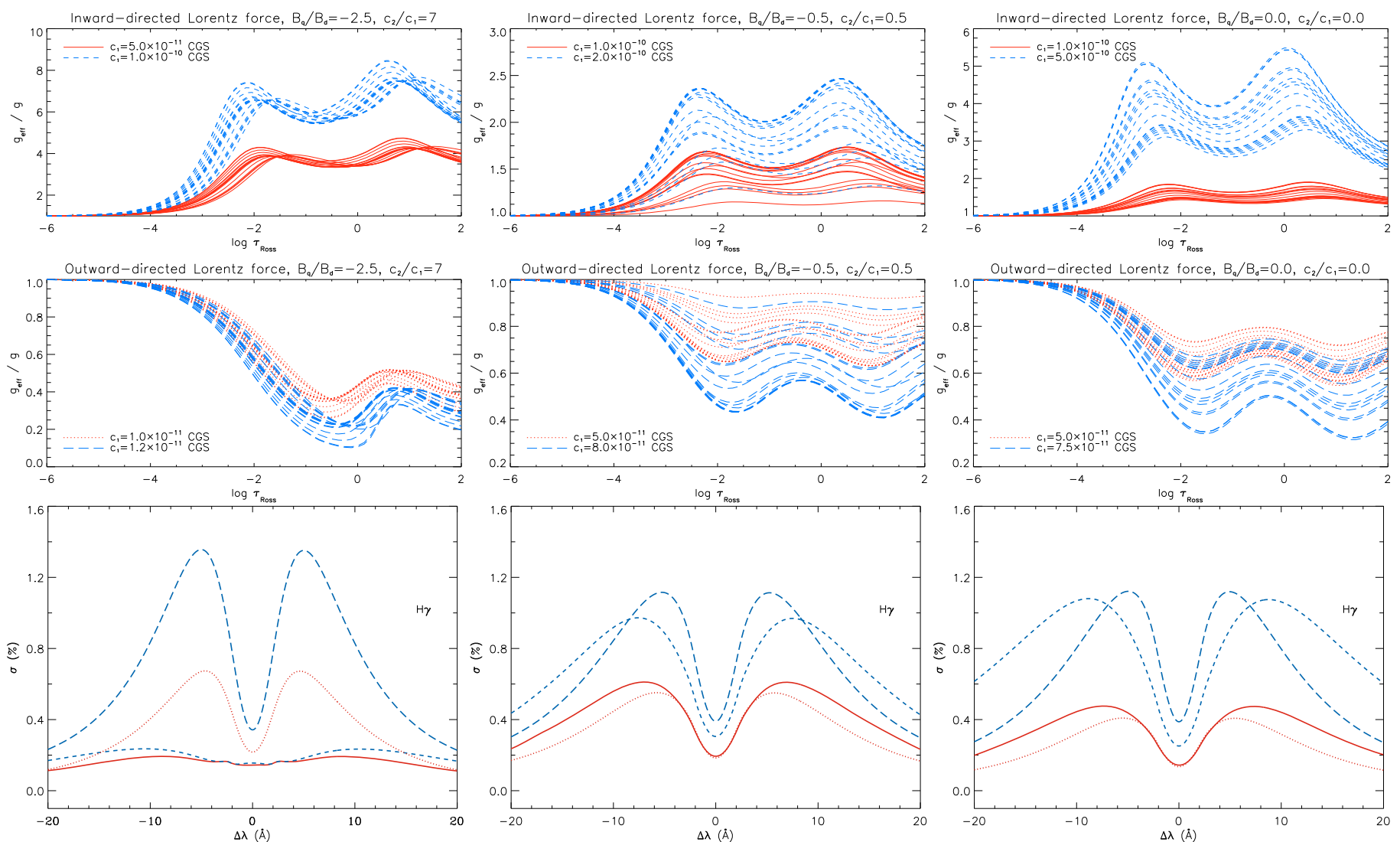

Fig. 5. The effective acceleration as a function of the Rosseland optical depth for different rotation phases calculated for several magnetic field configurations and induced e.m.f. The resulting standard deviations around $\mathrm{H} \gamma$ line are shown in the bottom panel.

the amplitude of standard deviation by about a factor of two or more.

The amplitude of the standard deviation around hydrogen lines strongly depends on the magnitude of the inward-directed Lorentz force for different magnetic field geometries. For instance, the middle panel of Fig. 5 illustrates standard deviations for the model with $B_{\mathrm{q}} / B_{\mathrm{d}}=-0.5$ : changing induced $e . m . f$. by a factor of two considerably increases the amplitude of the standard deviation. Similarly, the right panel of Fig. 5 shows the predicted variations for the purely dipolar model.

\subsection{Comparison with the observations}

In the following we compare the residual theoretical and observed Balmer lines with model predictions based on different assumptions about the magnetic field geometry and the direction of the Lorentz force. The residuals are obtained by subtracting a spectrum at a reference phase $(\phi=0.114$ where the Balmer profiles have the largest widths) from all the other spectra. Figure 7 illustrates residual $\mathrm{H} \alpha, \mathrm{H} \beta$, and $\mathrm{H} \gamma$ line profiles for each of the observed rotation phases. The positive sign of the residuals implies that the lines at the current phase are narrower than those obtained at the reference phase. It is seen that the characteristic behavior of hydrogen lines demonstrates a single wave variation with the most noticeable effect at phases between $\phi=0.402$ and $\phi=0.855$. The effect is also seen in the red wings of lines at $\phi=0.985$; however, it is smeared out in the blue wing. We emphasize that this systematic asymmetry, with the blue wing lying below the red one, is observed for all three studied Balmer lines. The inaccuracy in the spectrum processing can be one of the reasons for this effect. However, our data reduction is identical to the analyzis of $\theta$ Aur (see Shulyak et al. 2007), which shows no such asymmetry. Thus, we suspect that the asymmetry may also have a physical origin because of the non-stationary, magnetically-channeled stellar wind from the surface of 56 Ari. Very fast rotation and a relatively high temperature of this star make it plausible that its wind produces the variable, obscured PCyg feature distorting blue wings of the Balmer lines. The presence of a weak feature approximately $2.5 \AA$ blueward of the line center seen for all three hydrogen lines could also be an argument for the wind. (For $\mathrm{H} \gamma$ line the presence of this feature could alternatively be explained by absorption in Ti II and Fe II lines; however, in the case of $\mathrm{H} \beta$, there are no spectral lines that could contribute to this feature.) Despite these problems introduced by an unknown physical process, the characteristic shape of the Lorentz force induced variability is clearly seen in the hydrogen lines of 56 Ari, making it possible to perform the analyzis in the framework of our modeling approach.

None of the tested theoretical models appeared to fit the observed variability in all phases, but for more than half of them models still generally describe the data more or less reasonably well (see, for example, Models 1 or 4). Varying the magnetic field geometry and the ratio of induced dipolar and quadrupolar equatorial e.m.f.'s $\left(c_{2} / c_{1}\right)$, we could achieve good agreement only for a certain phase interval: either it was possible to fit the observations around phase $\phi=0.5$ or only for other phases. As an example, in Fig. 7 we plot some of the theoretical predictions for models that provide a more or less reasonable fit to the observations. In particular, models with the outward-directed 
D. Shulyak et al.: The Lorentz force in atmospheres of chemically peculiar stars: 56 Arietis
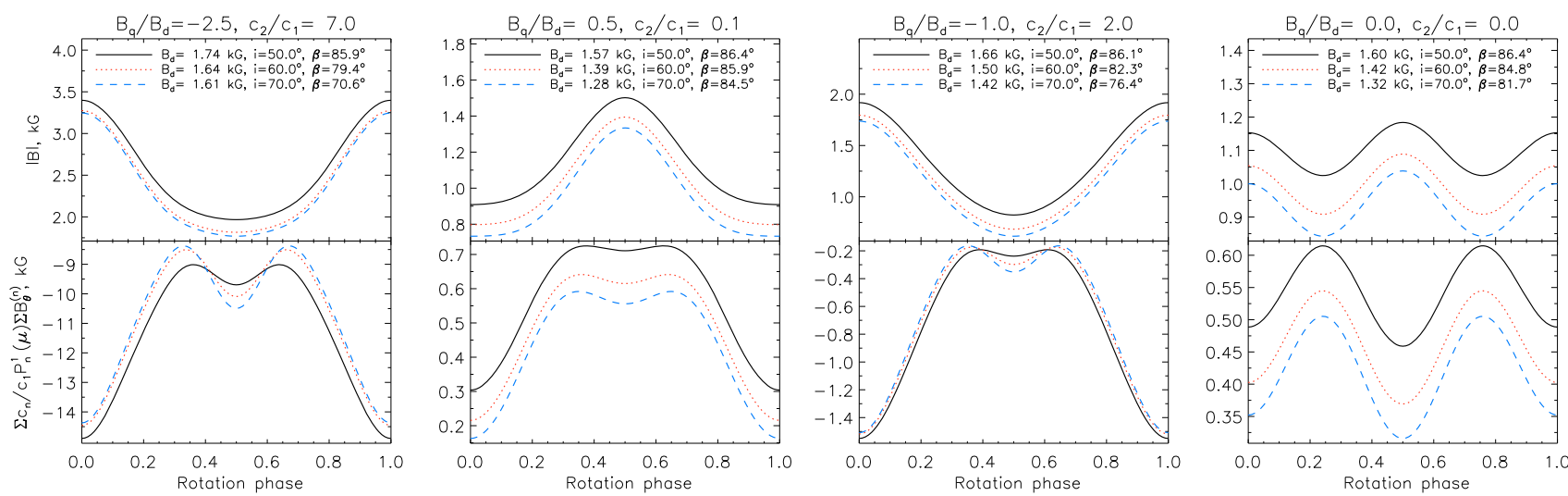

Fig. 6. Magnetic field modulus and Lorentz force parameter as a function of rotation phase for several magnetic field models.

Lorentz force are shown for the following configurations: $B_{\mathrm{q}} / B_{\mathrm{d}}=-2.5, c_{1}=1.2 \times 10^{-11} \mathrm{cgs}, c_{2} / c_{1}=7.0($ Model 1$)$,

$B_{\mathrm{q}} / B_{\mathrm{d}}=-4.5, c_{1}=1.2 \times 10^{-11} \mathrm{cgs}, c_{2} / c_{1}=7.0($ Model 2$)$,

$B_{\mathrm{q}} / B_{\mathrm{d}}=+0.5, c_{1}=9.0 \times 10^{-11} \mathrm{cgs}, c_{2} / c_{1}=0.1$ (Model 3).

Also, the model with the inward-directed Lorentz force is presented:

$B_{\mathrm{q}} / B_{\mathrm{d}}=-1.0, c_{1}=5 \times 10^{-10} \mathrm{cgs}, c_{2} / c_{1}=2$ (Model 4).

For all plotted models, the inclination angle $i=50^{\circ}$ was assumed. Taking $i=70^{\circ}$ does not change the disk-integrated parameters of the magnetic field much and thus leads to essentially the same picture of the hydrogen lines variation (see below).

Models 1 and 2 have the same parameters except for the strength of quadrupolar magnetic field component. They produce almost the same fit to the observed variations, and in the same manner, are not able to fit phases at $\phi=0.636$ and above (Although Model 2 systematically gives a little bit better fit there). Models with $B_{\mathrm{q}} / B_{\mathrm{d}}<-1.5$ give the same kind of the fit, but we do not plot them here to avoid overcrowding the plot. At the same time, Model 3 seems to be a preferable one for these phases, but it fails to fit observations at phases $\phi=0.435,0.478,0.502$, and gives an enormously strong effect at phases $\phi=0.321$ and down to zero. This is also true for Model 4 with the inward-directed Lorentz force. This model fits such phases as $\phi=0.689,0.708$ reasonably well, but yields the line wings that are generally too wide for comparing with observed ones. Thus, of the two possible directions of the Lorentz force in our model, we consider an outward-directed Lorentz force as the more reasonable choice to describe observations of 56 Ari. Because of problems with telluric lines, the continuum normalization around $\mathrm{H} \alpha$ line is substantially inaccurate comparing to other lines and it is not possible to distinguish between different models there.

By testing models with different magnetic field parameters, we tried to find those that predict a single-wave variation of the magnetic force term over the rotation cycle, as indicated by observations. Moreover, its run is likely to have a wide plateau around $\phi=0.5$ and drop rapidly close to $\phi=0$ and $\phi=1$ to fit observations (see Fig. 7). By varying the parameters $B_{\mathrm{q}} / B_{\mathrm{d}}$ and $c_{2} / c_{1}$ (with the fixed $i$ and $\beta$ ), we succeeded in finding sets of parameters that give this kind of plateau, but in all cases it appears to be not as wide as needed to fit observations in all phases. This is illustrated in Fig. 6 where we plot the magnetic parameters used for the Lorentz force calculation in some of the models mentioned above as a function of the rotation phase. The right panel in this figure illustrates predictions for the purely dipolar model. It is also seen that the inclination angle $i$ does not play a critical role in the present investigation: models with different $i$ would give the same phase-resolved variation in hydrogen lines, and any amplitude difference between them can be adjusted by a proper choice of a $c_{1}$ parameter.

In this investigation we focused analyzis on the hydrogen lines. It appears that they are most sensitive to the pressure changes introduced by the Lorentz force. As for metal lines, none of the strong Si II lines visible in the spectrum of 56 Ari, $\lambda 5055.98 \AA, 5466.48 \AA, 5466.89 \AA, 6347.11 \AA$ or $6371.37 \AA$, exhibits significant variation due to a non-zero Lorentz force. We have tested this by using the magnetic parameters of Model 1 (which has the largest amplitude of the $g_{\text {eff }}$ variation, see Fig. 5) and recomputing spectrum models for every phase with the mean abundances. We find no detectable changes in the line wings and less than a $1 \%$ difference in the line cores between models with and without Lorentz force. This difference is likely to stem from the differences in the temperature distribution of these two models. No visible phase-dependent changes can be seen in the spectra corresponding to the models with Lorentz force. These results led us to the conclusion that, because of their high pressure sensitivity in the predominantly ionized plasma of the atmosphere of such a relatively hot star, only the hydrogen lines are useful indicators for the magnetic pressure effects.

Similarly, we find no evidence of any phase-dependent pressure effects on the stellar spectral energy distribution. The maximum difference between models with and without the Lorentz force is less than $2 \%$ in Balmer continuum. This corresponds to $\mathrm{a} \approx 0.01$ mag difference in $c_{1}$ color-index and even less for other Strömgren parameters. Thus, variation seen, for example, in the phase-resolved spectrophotometric scans of 56 Ari published by Adelman (1983) could not be attributed to the Lorentz force effects, but are produced by inhomogeneous abundances and/or other mechanisms.

We note that the strong decrease in $g_{\text {eff }}$ evident in Fig. 5 (up to $\approx 1$ dex around $\log \left(\tau_{\text {Ross }}\right)=0$ ), compared to the non-magnetic case, leads to a relatively small difference in the observed parameters because of a) the fact that this decrease does not affect the entire stellar atmosphere and b) non-local nature of the hydrostatic equation in the presence of depth-dependent $g_{\text {eff }}$. The latter implies that, for example, one order of magnitude increase in the magnetic gravity only results in three times lower gas pressure for the outward-directed Lorentz force, which is too small to significantly change the opacity coefficient and influence the model structure. The difference between magnetic models for different rotational phases is even less since $g_{\text {eff }}$ varies maximum by a factor of $\approx 2$ for Model 1 .

Finally, we stress that it is difficult to conclude anything with certainty regarding the preferable model of the magnetic 

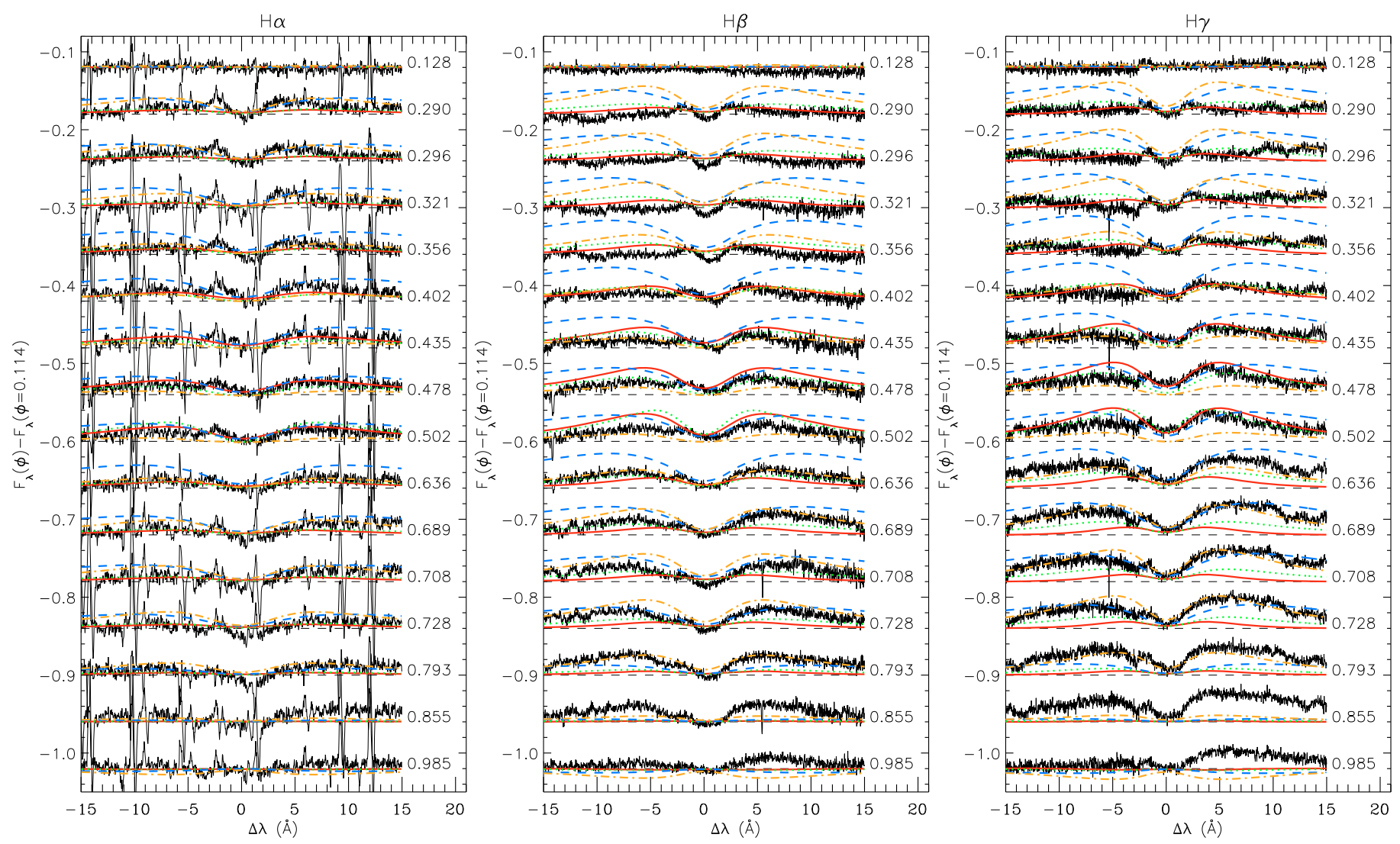

Fig. 7. Residual profiles of the $\mathrm{H} \alpha, \mathrm{H} \beta$, and $\mathrm{H} \gamma$ lines for each of the observed phase relative to the phase 0.114 . Thin solid line - observations. The theoretical profiles are shown for the following models: thick solid line $-B_{\mathrm{q}} / B_{\mathrm{d}}=-2.5, c_{2} / c_{1}=7$ model (outward-directed Lorentz force), dotted line $-B_{\mathrm{q}} / B_{\mathrm{d}}=-4.5, c_{2} / c_{1}=7$ model (outward-directed Lorentz force), dash-dotted line $-B_{\mathrm{q}} / B_{\mathrm{d}}=0.5, c_{2} / c_{1}=0.1$ model (outward-directed Lorentz force), dashed line $-B_{\mathrm{q}} / B_{\mathrm{d}}=-1.0, c_{2} / c_{1}=2$ model (inward-directed Lorentz force). The residual spectra for consecutive phases are shifted in the vertical direction. The thin dashed line gives the zero level for each spectrum.

field geometry without additional accurate magnetic observations of 56 Ari. Furthermore, other dynamic processes, such as Hall's currents and particle diffusion, may contribute to the observed variations in hydrogen lines. These processes cannot be accounted for in our modeling because of their complex nature. Nevertheless, similar to the results of the Paper I, in this study we demonstrate that the observations can be described with a simple geometrical approach under the assumption of strong surface electric currents in the atmosphere of a main-sequence mCP star.

\section{Conclusions}

With the use of the high-resolution, phase-resolved observations of a magnetic CP star 56 Ari and employing modern model atmosphere technique, we detected and investigated variations in the Stark-broadened profiles of $\mathrm{H} \alpha, \mathrm{H} \beta$, and $\mathrm{H} \gamma$ lines in the framework of a Lorentz force model. Several proofs of significant magnetic pressure in the atmosphere of 56 Ari have been found:

- The characteristic shape of the variation during a full rotation cycle of the star corresponds to those described by Kroll (1989) and other authors as a result of the impact of a substantial Lorentz force (see Paper I and references therein).

- Numerical calculations of the model atmospheres with individual abundances demonstrate that the surface chemical spots cannot produce the observed variability in the hydrogen line profiles of the star.
- Our model shows reasonable agreement with the observations if the outward-directed magnetic force is applied assuming the dipole+quadrupole magnetic field configuration. Unfortunately, large uncertainties in the available observations of the longitudinal magnetic field made it impossible to conclude anything confidently about the strengths of the quadrupolar component.

- Taking a variety of possible solutions into account, we find that, to fit the amplitude of a phase-resolved variation in $\mathrm{H} \alpha$, $\mathrm{H} \beta$, and $\mathrm{H} \gamma$ lines, the magnitude of an induced equatorial e.m.f. must be in the range $10^{-11}-10^{-10} \mathrm{cgs}$ in case of an outward-directed Lorentz force and $\sim 5 \times 10^{-10}$ in case of an inward-directed one.

\section{Discussion}

56 Ari is the second magnetic CP star for which we detected the characteristic variation in the hydrogen Balmer line profiles and performed detailed modeling of the Lorentz force effect. Our previous target, A0p star $\theta$ Aur, also demonstrated significant variability in the hydrogen Balmer lines (see Paper I). However, in the case of $\theta$ Aur, Borra \& Landstreet (1980) provided much more accurate measurements of the longitudinal magnetic field variations, which allowed us to determine geometry of its surface magnetic field more precisely. Nevertheless, for both stars we find that the outward-directed Lorentz force is needed to explain observations. However the induced e.m.f. for 56 Ari may differ by an order of magnitude, but this remains uncertain because of poorly known magnetic field geometry. 
A single-wave variation of the residual spectra is a characteristic signature for both $\theta$ Aur and 56 Ari. Since both stars have high inclination angles and magnetic obliquities, in the framework of our Lorentz force model this variation indicates the presence of a more complex magnetic field geometry than a simple dipole. Such a variation can be obtained in the nondipolar theoretical models by a proper choice of induced e.m.f. for each of the multipolar components (e.g. the $c_{2} / c_{1}$ ratio in the case of dipole+quadrupole combination). Furthermore, for both stars the amplitude of the longitudinal magnetic field variation is about $\approx 500 \mathrm{G}$, which can be the reason for the similar amplitude of the detected Balmer lines variation $(\approx 1 \%)$ since the effective temperatures of stars are different $\left(T_{\text {eff }}(\theta\right.$ Aur $)=10400 \mathrm{~K}$, $T_{\text {eff }}(56$ Ari $\left.)=12800 \mathrm{~K}\right)$. Similar to Paper I, we do not consider any details here of the physical mechanisms that could be responsible for the observed Lorentz force. The final conclusion about the nature of the significant magnetic pressure can only be obtained when more sophisticated models of the magnetic field evolution and its interaction with highly magnetized atmospheric structure have become available and/or alternative models been tested (however, for some of the estimates see discussion in Shulyak et al. 2007).

In the present work we made use of a simple geometrical 1D model of Lorentz force: the surface averaged values of the transverse magnetic field and the magnetic field modulus are introduced in the hydrostatic equation of the stellar matter. Future investigations can benefit from taking $2 \mathrm{D}$ effects into account with direct surface integration of the hydrogen line profiles computed with individual models. This could also probably open a possibility of accounting for the Hall's currents. Unfortunately, as mentioned above, this is difficult to do at present, but by no means impossible once more computational resources become available.

The dependence of the observed variability in hydrogen lines upon the magnetic field geometry and strength is one of the key elements in our investigation. If such a dependence exists, it could bring a number of theoretical constraints on the interaction of the magnetic field with stellar plasma. So far, we have analyzed only two stars with occasionally similar longitudinal magnetic field intensity. We are limited to stars for which the configuration of a magnetic field can be extracted from the literature and that can be observed with highly stable spectrometers like BOES to reduce possible errors in spectra processing. Thus, observations of other $\mathrm{mCP}$ stars are needed to conclude about the connection between magnetic field and variability seen in Balmer lines.
Acknowledgements. The authors are thankful to Tanya Ryabchikova for her help preparing of line lists used in DI. We also acknowledge the use of cluster facilities at the Institute of Astronomy, Vienna University. This work was supported by the FWF Lise Meitner grant Nr. M998-N16 to D.S. O.K. is a Royal Swedish Academy of Sciences Research Fellow supported by a grant from the Knut and Alice Wallenberg Foundation. Han acknowledges the support for this work from the Korea Foundation for International Cooperation of Science and Technology (KICOS) through grant No. 07-179. Based on INES data from the IUE satellite.

\section{References}

Adelman, S. J. 1983, A\&AS, 51, 511

Adelman, S. J., Malanushenko, V., Ryabchikova, T. A., \& Savanov, I. 2001, A\&A, 375, 982

Barklem, P. S., Piskunov, N., \& O’Mara, B. J. 2000, A\&A, 363, 1091

Bagnulo, S., Landi Degl'Innocenti, M., Landolfi, M., \& Mathys, G. 2002, A\&A, 394, 1023

Borra, E. F., \& Landstreet, J. D. 1980, ApJS, 42, 421

García-Gil, A., García López, R. J., Allende Prieto, C., \& Hubeny, I. 2005, ApJ, 623,460

Hatzes, A. P. 1993, Peculiar versus Normal Phenomena in A-type and Related Stars, 44, IAU Coll.,138, 258

Hauck, B., \& Mermilliod, M. 1998, A\&AS, 129, 431

Kim, K. M., Jang, J. G., Chun, M. Y., et al. 2000, Publication of the Korean Astronomical Society, 15S, 119 (in Korean)

Kim, Kang-Min, Han, Inwoo, Valyavin, Gennady, G., et al. 2007, PASP, 119, 1052

Khan, S., \& Shulyak, D. 2006a, A\&A, 448, 1153

Kochukhov, O., Drake, N. A., Piskunov, N., \& de la Reza, R. 2004, A\&A, 424, 935

Kochukhov, O., Khan, S., \& Shulyak, D. 2005, A\&A, 433, 671

Kochukhov, O. P. 2007, Physics of Magnetic Stars, 109

Kroll, R. 1989, Rev. Mex. Astron. Astrofis., 2, 194

Kupka, F., Piskunov, N., Ryabchikova, T. A., Stempels, H. C., \& Weiss, W. W. 1999, A\&AS, 138, 119

Landstreet, J. D. 2001, in Magnetic Fields Across Hertzsprung-Russell Diagram, ed. G. Mathys, S. K. Solanki, \& D. T. Wickramasinghe, ASP Conf. Ser., 248, 277

Lehmann, H., Tkachenko, A., Fraga, L., Tsymbal, V., \& Mkrtichian, D. E. 2007, A\&A, 471, 941

Lipski, Ł., \& Stępień, K. 2008, MNRAS, 385, 481

Nicolet, B. 1978, A\&AS, 34, 1

Piskunov, N. E., Kupka, F., Ryabchikova, T. A., Weiss, W. W., \& Jeffery, C. S. 1995, A\&AS, 112, 525

Ryabchikova, T. A. 2003, in Magnetic Fields in O, B and A Stars, ed. L. A. Balona, H. F. Henrichs, \& R. Medupe, ASP Conf. Ser., 305, 181

Shulyak, D., Tsymbal, V., Ryabchikova, T., Stütz Ch., \& Weiss, W. W. 2004, A\&A, 428, 993

Shulyak, D., Valyavin, G., Kochukhov, O., et al. 2007, A\&A, 464, 1089, Paper I

Valyavin, G., Kochukhov, O., \& Piskunov, N. 2004, A\&A, 420, 993

Valyavin, G., Kochukhov, O., Shulyak, D., et al. 2005, JKAS, 38, 283

van Leeuwen, F. 2007, A\&A, 474, 653

Wrubel, M. H. 1952, ApJ, 116, 291 